\title{
A Modal-Based Iterative Circuit Model for the Analysis of CRLH Leaky-Wave Antennas Comprising Periodically Loaded PPW
}

\author{
J. S. Gomez-Diaz ${ }^{1}$, Student Member, IEEE, A. Alvarez-Melcon ${ }^{1}$, Senior Member, IEEE \\ T. Bertuch ${ }^{2}$, Member, IEEE
}

\begin{abstract}
A novel modal-based iterative circuit model is described for the calculation of the complex propagation constant of mushroom-like parallel-plate composite right/left handed leakywave antennas (PPW CRLH LWAs). The conventional lossless CRLH unit cell circuit is modified in order to consider the electromagnetic coupling to free space through a slot. For this purpose, a slot equivalent radiative structure, based on phasedarray theory, is analyzed using a mode matching approach combined with Floquet's theorem. A direct correspondence between lumped elements and this radiative structure is found, leading to a frequency-dependent unit cell circuit model. A quickly converging iterative algorithm is then employed to determine the final element values of the unit cell. The proposed method is accurate, and it takes into account the structure physical dimensions. It also allows to obtain a balanced CRLH unit cell design without requiring any full-wave simulation, is several orders of magnitude faster than full-wave simulations, and provides a deep insight into the physics of the antenna radiation mechanism.
\end{abstract}

Index Terms-Composite Right/Left-Handed (CRLH) metamaterials, Leaky-Wave Antennas (LWAs), Modal Analysis (MA), Parallel-Plate Waveguides (PPW)

\section{INTRODUCTION}

$\mathbf{M}$ ETAMATERIAL (MTM) leaky-wave antennas (LWA) are designed to operate in their fundamental guided mode $(\nu=0)$ (e.g. [1], [2]), while conventional periodic LWAs mostly use their first space harmonic $(\nu=-1)$ [3]. Several types of MTM LWAs have been presented in literature. All antenna types are based on the same underlying design principle, which is the periodic loading of a host transmission line (TL) in such a way that the resulting TL becomes a socalled composite right/left-handed (CRLH) TL [1] or negative refractive index (NRI) TL [2] respectively. In connection with frequency scanning LWAs, the term "CRLH" seems more adequate for describing the behavior of the loaded TL and thus it will be used synonymously for the term "MTM" throughout this article.

The main distinctive feature of all MTM LWAs found in literature is the type of host TL used. Typical host TLs are microstrip (MS) lines [4], [5], coplanar waveguides (CPW) [6], or coplanar striplines (CPS) [7]. Also the metallo-dielectric surfaces of the mushroom-type [8] have been employed to generate LW radiation [9]. The type of host TL determines

\footnotetext{
${ }^{1}$ Technical University of Cartagena, Campus Muralla del Mar s/n, Cartagena, E-30202, Spain, E-mail: jsebastian_gomez@ono.com

2 Fraunhofer FHR, Neuenahrer Str. 20, 53343. Wachtberg, Germany, Email: thomas.bertuch@fhr.fraunhofer.de
}

the polarization of the radiated field. LWAs based on MS lines or CPWs generate transverse magnetic (TM) polarization whereas LWAs comprising CPS lines radiate transverse electric (TE) fields. The mushroom surfaces can produce both TM [1], [10] and TE [9] polarization depending on the kind of excitation.

In order to analyze these types of antennas, circuit models are usually employed [1], [2]. These models are able to accurately represent the antenna dispersive behavior [i.e. the propagation constant $\beta(\omega)$ ] but they have difficulties to characterize the amount of radiated power [i.e. leaky factor $\alpha(\omega)$ ]. Therefore, the radiation characteristic of the antenna cannot completely be determined with these methods. This is an important limitation of the existing techniques, since then the attenuation factor cannot be controlled in the design of antennas for practical applications. In addition, a considerable number of time-consuming full-wave simulations are usually required for the design of CRLH LWAs. This makes the CRLH LWA design procedure a tedious task. Moreover, full-wave analysis does not provide any deep insight into the physics of the radiation phenomena, which is extremely important to understand and to speed-up the design process.

In this paper, a novel circuit model is employed for the analysis of mushroom-like CRLH LWAs comprising periodically loaded parallel-plate waveguides [11]. The elements of the circuit model are determined by an iterative algorithm combined with modal analysis. Specifically, the attenuation factor of the antenna is rigorously computed for the first time using an equivalent radiating structure, which is based on phased-array theory. The analysis of this structure leads to the accurate definition of a frequency-dependent circuit model, which relates the radiation characteristics with the physical dimensions of the antenna. An iterative algorithm is proposed to determine the values of the equivalent circuit. The main advantage of the method is that it models and describes in a simple way the complex CRLH LWA radiation phenomena using equivalent dispersive circuits. Furthermore, the proposed approach also serves to compute the physical dimensions of a balanced CRLH unit cell design. Note that the proposed technique is accurate and very efficient, requiring just minutes to analyze a complete LWA.

The paper is organized as follows. Sec. II describes the CRLH LWA comprising the loaded PPW under analysis. Sec. III presents the circuit model employed for the antenna characterization, and shows how the antenna complex propa- 


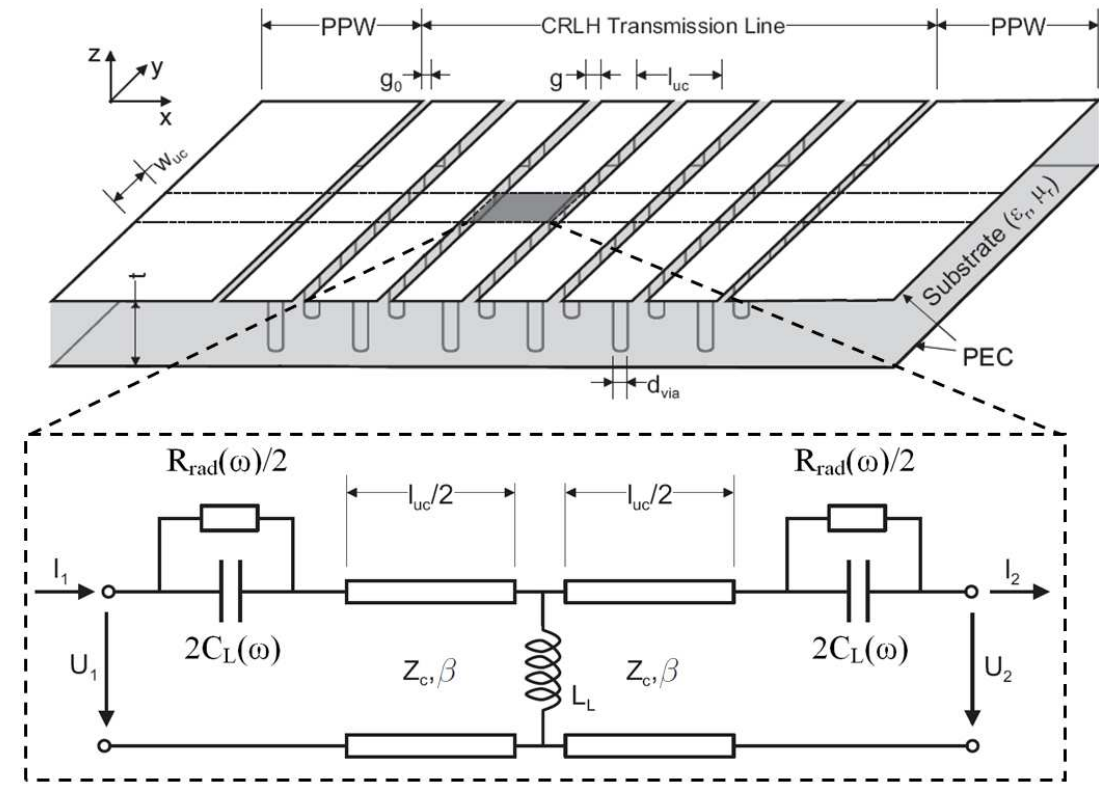

Fig. 1: Topology of a CRLH LWA comprising a periodically loaded PPW (top) and equivalent circuit model (bottom) representing a unit cell of the periodic one-dimensional CRLH TL. The loading is obtained by wires and slots. The slots also provide the coupling to free space, which is rigorously modeled by the dispersive lumped elements $C_{L}(\omega)$ and $R_{\text {rad }}(\omega)$.

gation constant can be derived from the equivalent circuit. In Sec. IV, a mode-matching analysis approach combined with Floquet's theorem is employed to analyze a slot-equivalent radiating structure, leading to a frequency-dependent lumped circuit model for the CRLH LWA unit cell. The dispersive values of this model are obtained using a quickly converging iterative algorithm, presented in Sec. V. In Sec. VI, the proposed method is employed to design and analyze a single CRLH unit cell first, and then a ten unit cell LWA. Full-wave simulations are included to completely validate the proposed technique. Finally, conclusions are given in Sec. VII.

\section{CRLH LWA COMPRISING LOADED PPW}

The top of Fig. 1 shows the topology of the proposed CRLH LWA including two laterally attached PPW feeding sections. At the bottom an equivalent circuit for the unit cell of the LWA's CRLH TL section is given. It will be discussed in the next section.

The structure is assumed to be finite in the $x$ - and $z$ directions and infinitely periodical in the $y$-direction. Effective wave propagation occurs in the $x z$-plane. The LWA consists of a planar substrate layer of thickness $t$ with homogeneous and isotropic material characterized by relative permittivity $\varepsilon_{r}$ and relative permeability $\mu_{r}$. Its back side is completely metalized. The front side is also metalized except for a finite number of parallel and equidistant slots. The first and last slots, used for matching to the input/output ports, have a width of $g_{0}$, whereas all other slots have the width $g$. The metal strips oriented along the $y$-direction between adjacent slots are connected to the back side metalization by a rectangular grid of metalized via holes with diameter $d_{v i a}$. The via holes are placed symmetrically in the center between adjacent slots with a spacing of $l_{u c}$ and $w_{u c}$ along the $x$ - and $y$-directions respectively. The vias and the slots constitute the loading of the PPW and when operated in the proper frequency band the loaded section behaves as a CRLH TL. Note that this CRLH line is attached to two conventional right-handed PPW at its ends, which constitute the antenna feeding and matching load. Without any slots present, the rectangular grid of via holes creates a so-called artificial dielectric (AD) or wire medium (WM) which exhibits strongly dispersive properties, similar to the ones observed in hollow cylindrical waveguides, including a cut-off frequency $f_{c, W M}$. In the past, open slabs of $\mathrm{AD}$ material have been used extensively to create forward scanning LWAs [12], [13], [14], [15]. The AD medium's dispersive properties are fundamental for the operation of the proposed CRLH LWA. If the distance $t$ between the metal planes is sufficiently small that only the fundamental parallel plate mode (PPM) can propagate in the unloaded PPW, the WM acts like a high pass filter. The WM's cut-off frequency $f_{c, W M}$ can be computed resorting to derivations given in [16]. Below cut-off, there is no propagation in the WM and above cut-off the WM supports $\mathrm{RH}$ propagation with an effective wave number along the $x$-direction that is always smaller than the free space wave number $k_{0}$.

Introducing the slots in the upper metalization has two effects. On the one hand, a coupling between the region above and the region inside the PPW is established, and on the other hand, a CRLH TL is created which may support lefthanded $(\mathrm{LH})$ propagation below the WM's cut-off frequency. Coupling of the regions facilitates leaky-wave (LW) radiation as long as the magnitude of the effective wave number's real part of the CRLH TL is smaller than the free space wave number $k_{0}$. At frequencies above $f_{c, W M}$ the propagating mode in the loaded PPW will be RH and thus, forward LW radiation will be observed. Depending on the geometry, 
below $f_{c, W M}$ the loaded PPW may support LH propagation which will result in backward LW radiation. If the CRLH TL exhibits the so-called "balanced" behavior [1], a smooth transition from left-handed to right-handed frequency regions is possible, as frequency varies. However, even in the case that the structure is completely balanced, the antenna presents a reduction in the radiation efficiency at broadside. This is because the PPW loading only provides a series resistor in the unit cell equivalent circuit, representing radiation losses, whereas a shunt resistor is also required to efficiently radiate at broadside [17], [18].

\section{Equivalent Circuit Model}

The equivalent circuit model related to a single unit cell (with a length $l_{u c}$ and a width $w_{u c}$ ) of the CRLH LWA is shown in Fig. 1 (bottom). The layout of the equivalent circuit assumes a symmetric composition of the unit cell along the direction of wave propagation. Two right-handed TLs, of length $l_{u c} / 2$, have been employed to model the PPW behavior (i.e. the host TL). These TLs are described by their characteristic impedance $Z_{c}$ and propagation constant $\beta$. It is very important to distinguish between $\beta$ and $k_{\text {eff }}$. The former is related to the host TL (unloaded PPW) and it is typically real, as long as material losses are neglected. The latter is related to the total CRLH unit cell and it is complex, because it also includes the radiations losses of the structure. The LH behavior is achieved by a via-hole and by two halfslots (see Fig. 1). The loading is modeled in the equivalent circuit with a shunt inductance $\left(L_{L}\right)$ and two symmetrically placed dispersive circuit elements, composed of the parallel connection of a resistor $\left[R_{\text {rad }}(\omega)\right]$ and a capacitor $\left[C_{L}(\omega)\right]$. The parallel connection of the two elements is convenient to represent the radiation mechanism through the slot. In the limiting case of a narrow slot, the capacitor becomes very large, and tends to reduce the radiation by short-circuiting the resistance. This correctly models the radiation reduction that occurs in the real structure for very narrow slots. Note that this dispersive circuit rigorously takes into account the effects of the slot, including the physical parameters of the structure, coupling to free-space, reactive fields, coupling to other slots, radiation losses, and the capacitive behavior required to balance the unit cell. An equivalent radiating structure and the modal analysis employed to derive the values of the equivalent circuit elements, including dispersion will be explained in the following section.

In order to compute the complex propagation constant of the CRLH unit cell, we represent the equivalent circuit in terms of transmission matrices. This helps to obtain the value of the shunt inductance $L_{L}$ for the given geometry, and to determine the complex propagation constant of the unit cell. In the next discussion it is assumed that the physical dimensions of the CRLH unit cell are known. In the following sections, we will explain how to accurately obtain these physical dimensions, without the need to use full-wave simulations.

The first step required for the analysis is to obtain the characteristic impedance and the propagation constant of the unloaded PPW, related to a single unit cell of length $\ell_{u c}$ and

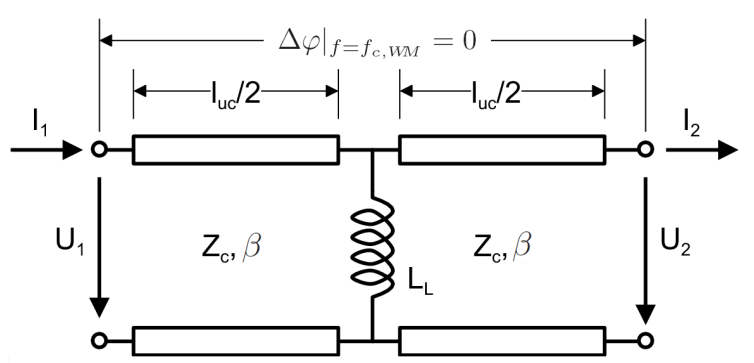

Fig. 2: Equivalent circuit model of a unit cell related to a PPW loaded by a periodic grid of wires. The circuit model of Fig. 1 reduces to this model at the CRLH TL transition frequency [1], assuming that the cell is balanced.

width $w_{u c}$. These values may be obtained as

$$
\begin{aligned}
Z_{c} & =\frac{t}{w_{u c}} \sqrt{\frac{\mu_{r} \mu_{0}}{\varepsilon_{r} \varepsilon_{0}}}, \\
\beta & =\omega \sqrt{\varepsilon_{r} \varepsilon_{0} \mu_{r} \mu_{0}},
\end{aligned}
$$

where $\varepsilon_{0}$ and $\mu_{0}$ are the permittivity and the permeability of vacuum respectively, and $\omega$ is the angular frequency. Then, the transmission matrix of the host TL of length $\ell_{u c} / 2$ may be expressed as

$\underline{\underline{T}}_{\text {host }}=$

$1\left(e^{j \beta l_{u c} / 2}+e^{-j \beta l_{u c} / 2}\right.$

$\overline{2}\left(1 / Z_{c}\left(e^{j \beta l_{u c} / 2}-e^{-j \beta l_{u c} / 2}\right)\right.$

$\left.\begin{array}{c}Z_{c}\left(e^{j \beta l_{u c} / 2}-e^{-j \beta l_{u c} / 2}\right) \\ e^{j \beta l_{u c} / 2}+e^{-j \beta l_{u c} / 2}\end{array}\right)$.

Next, the PPW loaded by a grid of via-holes is considered. This creates an artificial dielectric with strong dispersive properties. Similar to hollow waveguides, where the metallic side walls introduce the same effect, the AD acts like a high pass on the fundamental PPM. The cut-off frequency $f_{c, W M}$ of the $\mathrm{AD}$ can be found by solving (e.g. numerically) the following dispersion equation (see [16])

$$
k w_{u c} \tan \left(\frac{k w_{u c}}{2}\right)=\frac{\pi w_{u c}}{l_{u c} \ln \left(\frac{l_{u c}}{\pi d_{v i a}}\right)},
$$

where $k=2 \pi f_{c, W M} \sqrt{\varepsilon_{r} \varepsilon_{0} \mu_{r} \mu_{0}}$ is the intrinsic wave number of the substrate material. Note that the effective wavelength in the $\mathrm{AD}$ becomes infinite at the cut-off frequency, which means that the propagation constant tends to zero. Therefore, this frequency corresponds to the transition frequency of a CRLH TL.

The transmission matrix of the shunt inductance is given by

$$
\underline{\underline{T}}_{L}=\left(\begin{array}{cc}
1 & 0 \\
\frac{1}{j \omega L_{L}} & 1
\end{array}\right) \text {. }
$$

In order to determine the value of the inductance, we will analyze the CRLH unit cell at the transition frequency. At this frequency, the phase shift at the ports of the unit cell becomes zero, and the model of Fig. 1 (bottom) reduces to the circuit of Fig. 2, as is demonstrated in [1]. The boundary conditions applied to the currents $\left(I_{1}, I_{2}\right)$ and the voltages $\left(U_{1}, U_{2}\right)$ may be formulated as

$$
\left(\begin{array}{c}
U_{1} \\
I_{1}
\end{array}\right)=\underline{\underline{T}}_{h o s t} \underline{\underline{T}}_{L} \underline{\underline{T}}_{h o s t}\left(\begin{array}{c}
U_{2} \\
I_{2}
\end{array}\right)=\left(\begin{array}{c}
U_{2} \\
I_{2}
\end{array}\right)
$$




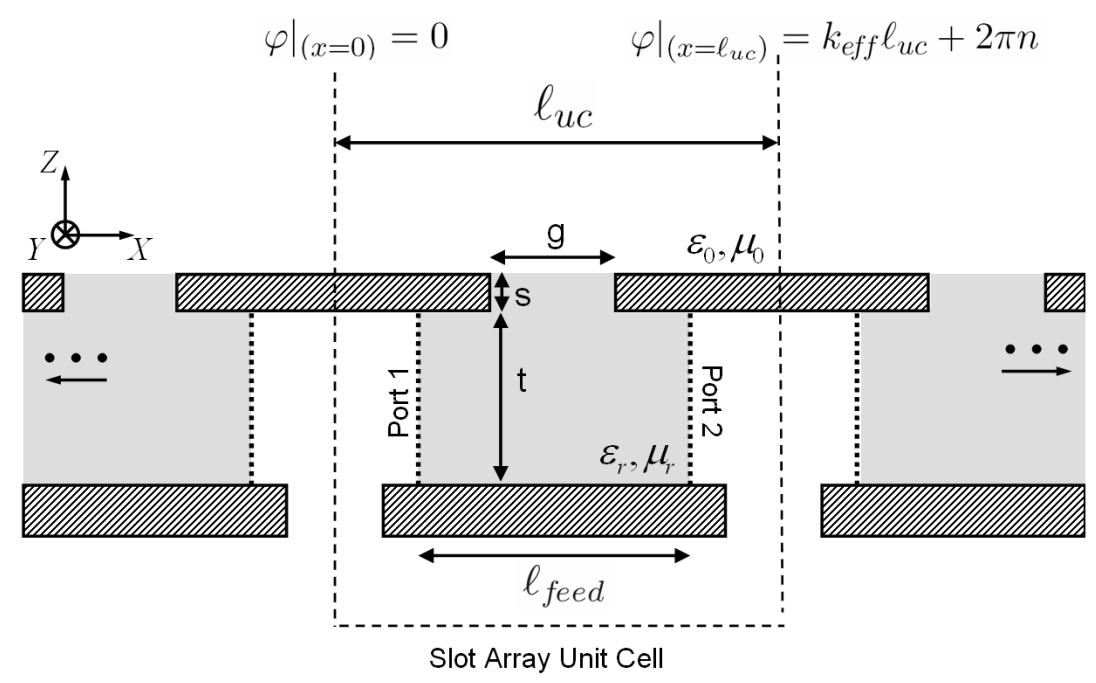

Fig. 3: Cross-section of one-dimensional periodic array of infinitely long slots radiating into free space, employed to rigorously model the CRLH LWA radiation mechanism. Periodic boundary conditions in free space are imposed at the limits of the unit cell. Each slot is attached to a PPW T-junction with two PPW ports. Port 1 serves as excitation of the array element.

and $L_{L}$ is determined by solving

$$
\operatorname{det}\left(\underline{\underline{T}}_{h o s t} \underline{\underline{T}}_{L} \underline{\underline{T}}_{h o s t}-\underline{\underline{I}}\right)=0
$$

where $\underline{\underline{I}}$ is the unitary matrix.

The transmission matrix which models the slot in the upper metalization of the host TL is represented by $\underline{\underline{T}}_{\text {gap }}$, which will be derived in the next section. The behavior of half of a slot (required to maintain the unit cell symmetry) is obtained as the square root of the $\underline{\underline{T}}_{\text {gap }}$ matrix, and it is denoted by $\underline{\underline{T}}_{\text {gap } / 2}$.

The transmission matrix associated to the total CRLH unit cell $\left(\underline{\underline{T}}_{u c}\right)$ is then obtained by a simply multiplication of the transmission matrices related to the unit cell elements, as follows

$$
\begin{aligned}
\underline{\underline{T}}_{u c} & =\underline{\underline{T}}_{g a p / 2} \underline{\underline{T}}_{\text {line }} \underline{\underline{T}}_{L} \underline{\underline{T}}_{\text {line }} \underline{\underline{T}}_{\text {gap } / 2} \\
& =\left(\begin{array}{ll}
A_{u c} & B_{u c} \\
C_{u c} & A_{u c}
\end{array}\right) .
\end{aligned}
$$

Note that the diagonal elements of $\underline{\underline{T}}_{u c}$ are identical, due to the equivalent circuit symmetry.

The complex propagation constant $\left(k_{e f f}\right)$ related to the total unit cell may be then determined by solving

$$
\operatorname{det}\left(\underline{\underline{T}}_{u c}-e^{j k_{e f f} l_{u c}} \underline{\underline{I}}\right)=0,
$$

which yields the complex value of

$$
k_{e f f}=\frac{\ln \left(A_{u c} \pm \sqrt{B_{u c} C_{u c}}\right)}{j l_{u c}} .
$$

Finally, note that the complex propagation constant $\left(k_{e f f}\right)$ can also be obtained using alternatives approaches (such as the described in [19]), once the different transmission matrixes employed in the analysis are known.

\section{EQUivalent RADiating Structure}

In this section, a rigorous computation of the transmission matrix $\underline{\underline{T}}_{\text {gap }}$, which characterizes the unit cell slot behavior, is presented. For this purpose, an equivalent phased-array antenna model is employed. It consists of a one-dimensional periodic array of infinitely long slots in a metal plane. At this point, we assume an infinite number of elements (slots) along the $x$-direction. This assumption is not critical for the analysis of leaky-wave antennas [3], since they are usually several wavelengths long. Using phased-array theory, we assume that all array elements are fed with a progressive phase shift. Each slot is individually attached to a T-junction formed with the PPWs, as shown in Fig. 3. In the figure, the horizontal PPWs which feed the slots are not interconnected, in order to clearly identify the different unit-cells. The total length of the whole feeding PPW is $\ell_{\text {feed }}$, which must be greater than $g$. Note that the influence of this TL will be removed at the end, in order to characterize an isolated slot in an external array environment. Due to this, it is sufficient to consider a single unit cell (array element) with imposed periodic boundary conditions in free space along the $x$-direction (see Fig. 3). Moreover, the imposed phase shift at a given frequency is determined by the effective wave number of the CRLH TL unit cell as $\Delta \varphi=k_{\text {eff }} l_{u c}+2 \pi n$.

Then, the single array element is studied using a multi mode-matching (MM) approach [20] combined with Floquet's theorem. The reason to use a multi-mode analysis is that not only propagative modes, but also evanescent modes must be rigorously taken into account. This is especially important to model the coupling from the PPW to the slot, from where the energy is radiated.

In order to perform the analysis, the equivalent radiating structure of Fig. 3 is split into a PPW E-plane T-junction (see Fig. 4) and into a slot fed by a vertical PPW (see Fig. 5). Then, the general scattering matrix (GSM) [21] associated to each individual structure $(\underline{\underline{G S M}}$ Tjunction and $\underline{\underline{G S M}}$ Aperture $)$ is obtained by using mode-matching techniques [20]. Next, both GSMs are combined into a single matrix $\left(\underline{\underline{G S M}}_{\text {gap }}\right)$, related to the total radiating array element. Note that the radiation 


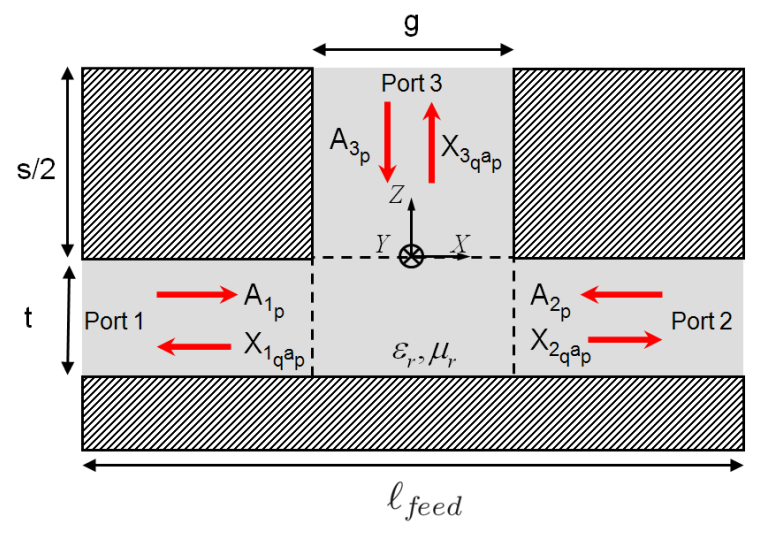

Fig. 4: Cross-section of an E-plane T-junction of parallel-plate waveguides.

mechanism of the structure is embedded into $\underline{\underline{G S M}}_{\text {gap }}$. At this point, this matrix is further simplified, considering only the fundamental PPW mode. This approximation is accurate, because although evanescent modes couple to the slot and have strong influence on the radiation, they are strongly attenuated as they propagate down the ports. In this way we obtain a matrix $\underline{\underline{S}}_{\text {gap }}^{\prime}$, which contains the scattering parameters related to the total radiating array element. However, we are interested only in modeling the slot. Consequently, we deembed the reference planes of the ports to the plane $x=\ell_{\text {feed }} / 2$ (as shown in Fig. 3), resulting into the scattering matrix $\underline{\underline{S}}_{\text {gap }}$. Finally, we perform a simple transformation from the $\underline{\underline{S}}_{\text {gap }}$ matrix to the transmission matrix $\underline{\underline{T}}_{\text {gap }}$ [21].

It can be expected that the magnetic field inside the slots and above the slots in Fig. 1 will be primarily polarized parallel to them. Hence, it will be sufficient, in the following modal analysis to consider TM waves. The reference directions of these waves change as a function of the PPW orientation (from $x$ to $z$-direction according to Fig. 4).

The steps to perform the analysis described above are detailed in the next subsections, including a validation of the approach using full-wave simulations.

\section{A. Modal Analysis of a PPW E-plane T-junction}

The E-plane T-junction in a parallel-plate waveguide has extensively been studied in the past [22], [23], [24]. A general cross-section of this junction is depicted in Fig. 4. In order to build the GSM associated to it, we individually excite each port of the junction with an incident TM mode denoted by $A_{a_{p}}$ (where $a \in\{1,2,3\}$ is the incident port number and $p=$ $1 \ldots m$ is the mode number). Then, we obtain the complex mode amplitudes $\left(X_{b_{q} a_{p}}\right.$, where $b \in\{1,2,3\}$ is the observation port and $q=1 \ldots m$ is the observation mode) using the analytic series solution method proposed in [23]. Note that the modal coefficients are referred to the T-junction borders (dashed line in Fig. 4), and that these coefficients directly correspond to the generalized scattering parameters. Then, the exact length of the T-junction ports are taken into account by moving the reference plane of each modal coefficient, using

$$
S_{b_{q} a_{p}}=X_{b_{q} a_{p}} e^{j\left(k_{a_{p}} \ell_{a}+k_{b_{q}} \ell_{b}\right)}
$$

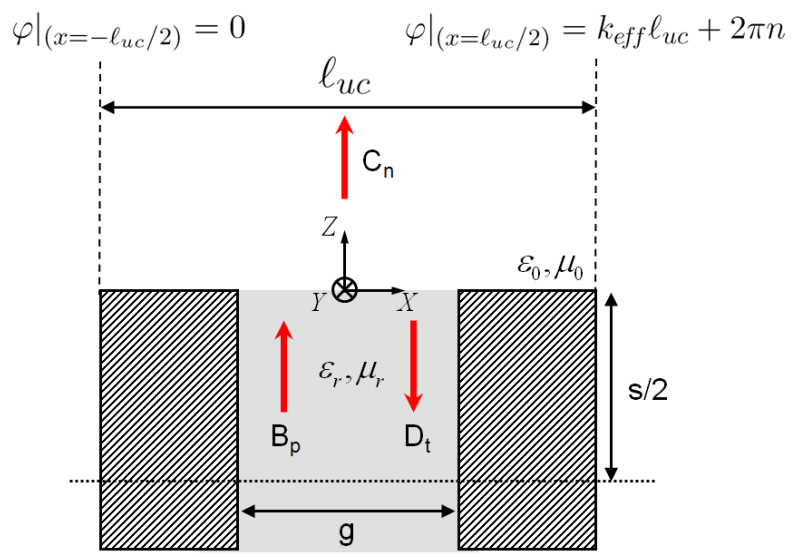

Fig. 5: Cross-section of an open-ended parallel-plate waveguide radiating in an array environment. Periodic boundary conditions, related to the complex propagation constant of the complete CRLH LWA unit cell, are imposed in the free-space region.

where $S_{b_{q} a_{p}}$ is a complex mode amplitude related to the origin of the observation port $b, \ell_{a}$ and $\ell_{b}$ are the lengths of the waveguide sections related to ports $a$ and $b$ (which corresponds to $\ell_{\text {feed }} / 2-g / 2$ in the case of ports 1 and 2 and to $s / 2$ in the case of port 3 , as shown in Fig. 4 ), and $k_{a_{p}}$ and $k_{b_{q}}$ are the mode wavenumbers.

\section{B. Modal Analysis of Open-Ended PPW Array}

The study of an array of dielectric-filled waveguides radiating into free space has already been performed in the past [20], [25]. The structure is shown in Fig. 5, including periodic boundary conditions for the free-space radiation. Its simple geometry allows to perform a modal analysis, resorting to the procedures described in [20] and combined with Floquet's theorem.

The field within the vertical PPW in Fig. 5 is expanded in mode functions TM to $x\left(T M_{x}\right)$. This means that the electric vector potential is zero and the magnetic vector potential is

$$
\underline{A}_{P P W}=\underline{\hat{x}} \psi_{P P W},
$$

where $\underline{\hat{x}}$ is the unit vector in the $x$-direction and $\psi_{P P W}$ is the scalar wave potential, which is given by

$$
\begin{aligned}
\psi_{P P W}= & k_{w}^{-1} B_{p} \cos \left[k_{x, p}\left(x+\frac{g}{2}\right)\right] e^{-j k_{z, p}^{\prime} z}+ \\
& k_{w}^{-1} \sum_{t=0}^{\infty} D_{t} \cos \left[k_{x, t}\left(x+\frac{g}{2}\right)\right] e^{j k_{z, t}^{\prime} z}
\end{aligned}
$$

Here, $k_{w}$ is the intrinsic wave number of the filling material, $k_{x, p}=p \frac{\pi}{g}$, and the $z$-directed wave number as determined by the separability condition is

$$
k_{z, t}^{\prime}= \pm \sqrt{k_{w}^{2}-\left(t \frac{\pi}{g}\right)^{2}} .
$$

In (13), we excite the waveguide with the $p$-th mode, which has an amplitude $B_{p}$. This mode propagates along the waveguide until it reaches the aperture. There, some energy is reflected 
back towards the waveguide. This field is expanded into an infinite series of modes with complex amplitudes $D_{t}$ $(t=1 \ldots m)$, as indicated in Eq. (13). Furthermore, some energy is coupled to free space. In this region, the field is expanded in mode functions TM to $z\left(T M_{z}\right)$ which implies again that the electric vector potential is zero. Note that the propagation direction has been changed from $x$ - to the $z$-axis. In this situation the magnetic vector potential yields

$$
\underline{A}_{0}=\underline{\hat{z}} \psi_{0},
$$

with

$$
\psi_{0}=k_{0}^{-1} \sum_{n=-\infty}^{\infty} C_{n} e^{-j\left(k_{e f f}+n \frac{2 \pi}{l_{u c}}\right) x} e^{-j k_{z, n} z}
$$

and

$$
k_{z, n}= \pm \sqrt{k_{0}^{2}-\left(k_{e f f}+n \frac{2 \pi}{l_{u c}}\right)^{2}},
$$

where $k_{0}$ is the intrinsic wave number of free space and $k_{\text {eff }}$ is the effective wave number related to the CRLH TL unit cell as determined by Eq. (10). It is important to remark that Floquet's theorem has been employed in Eq. (16), because of periodicity, leading to a discrete set of complex modes $C_{n}$ instead of the usual continuous spectrum obtained in the single slot case [20]. Also, note that the imposition of the continuity of the electromagnetic field components across the boundary will assure that the slot radiation mechanism entirely depends on the complex propagation constant of the CRLH TL unit cell $k_{\text {eff }}$. This allows us to establish a fundamental relationship between the CRLH TL unit cell and the modal analysis performed of the equivalent radiating structure, which are closely inter-related.

For the analysis of the T-junction in Fig. 4 the propagation direction is the $x$-axis for ports 1 and 2 , and the modal expansion used is based on the traditional $T M_{x}$ modes with respect to the propagation direction. For port 3, the propagation direction is changed along the $z$-axis, but the modal expansion used in this region is still based on modes $T M_{x}$ for a correct matching of the fields. Therefore, in the parallel plate region of Fig. 5 these modes are hybrid with respect to the new propagation direction along- $z$.

With the help of [20], the components of the electromagnetic fields within the PPW and in the free space region are calculated from Eq. (12) and (15). Applying boundary conditions for the $x$ - and $y$-components of the fields in the plane $z=0$, and utilizing the orthogonality properties of the harmonic functions involved in the formulation for the scalar wave potentials, expressions for the modal amplitudes $D_{t}$ and $C_{n}$ are derived as a function of the excitation $B_{p}$. Finally, the generalized scattering matrix $\left(\underline{\underline{G S M}}{ }_{\text {Aperture }}\right)$ for the structure of Fig. 5 is obtained after performing a modal analysis for each incident mode.

\section{Analysis of the Total Equivalent Structure}

The equivalent radiating structure shown in Fig. 3 can now easily be modeled using the generalized scattering matrices

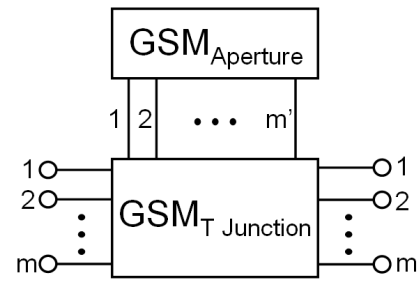

(a)

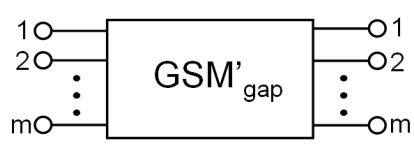

(b)
Fig. 6: Representation of the equivalent radiating structure of Fig. 3 using generalized scattering matrices (GSM). (a) Using the GSM related to the T-junction (see Fig. 4) combined with the GSM related to the aperture (see Fig. 5). (b) Using a single equivalent GSM.

$\underline{\underline{G S M}}_{T_{\text {junction }}}$ and $\underline{\underline{G S M}}$ Aperture , which are connected as indicated in Fig. 6(a). This connection can be further simplified, leading to a single matrix $\underline{\underline{G S}}_{\text {gap }}^{\prime}$ [see Fig. 6(b)].

In order to derive this ${\underline{G S M^{\prime}}}_{a p}$, a matrix formulation is developed. Specifically, boundary conditions are applied at the matrices' interconnection. The outgoing waves from the third port of the T-junction are considered the input waves to the aperture waveguide, meanwhile the reflected waves from the slot, due to the air discontinuity, are treated as the exciting waves at the T-junction's third port. Then, the input and output waves of $\underline{\underline{G S M^{\prime}}}$ gap are expressed as a function of all components of $\underline{\underline{G S M}}_{\text {junction }}^{\text {gap }}$ and $\underline{\underline{G S M}}_{\text {Aperture }}$. Therefore, the whole behavior of these two matrices is embedded into the matrix $\underline{\underline{G S}}_{\text {gap }}^{\prime}$ shown in Fig. 6(b).

In addition, the ${\underline{G S M^{\prime}}}_{\text {gap }}$ matrix is further simplified. Specifically, all higher order modes are neglected and only the fundamental PPW mode is considered. Note that the higher order modes have rigorously been taken into account to model the coupling from the T-junction to the slot, and to model the aperture radiation. However, since they are evanescent, they are strongly attenuated while propagating down the ports, and their contributions can be neglected. Therefore, the equivalent radiating structure may now be represented by a simple $(2 \times 2)$ scattering matrix relating the fundamental modes at the two ports $\left(\underline{\underline{S}}_{\text {gap }}^{\prime}\right)$.

At this point, it is important to remember that the goal is to model the effect of the slot in the PPW (including its radiation characteristics in a periodic environment) in order to be included into the CRLH unit cell model of Fig. 1 (bottom). Examining that model, one realizes that the effect of the host parallel-plate waveguide has already been considered. Therefore, we need to shift the reference plane of the last scattering matrix to the position of the slot, which may easily be done as

$$
\underline{S}_{b a}=\underline{S}_{b a}^{\prime} e^{-j \beta \ell_{f e e d}},
$$

where $a, b$ are the port numbers and $\beta$ and $\ell_{\text {feed }}$ are the propagation constant of the fundamental mode and physical length related to the feeding parallel-plate waveguide, respectively. This matrix is then transformed into the transmission matrix 
$\underline{\underline{T}}_{\text {gap }}[26]$, which may be expressed as

$$
\underline{\underline{T}}_{\text {gap }}=\left(\begin{array}{ll}
T_{11} & T_{12} \\
T_{21} & T_{22}
\end{array}\right)
$$

In this last transformation, the PPW characteristic impedance [see Eq. (1)] has been employed as a reference impedance [26]. This normalizes the resulting transmission matrix with respect to the unit cell width $\left(w_{u c}\right)$.

It is important to note that $\underline{\underline{T}}_{\text {gap }}$ models the entire radiation mechanism of the equivalent structure (see Fig. 3), including radiation losses, coupling to free space, reactive fields, coupling to other slots, and the slot influence within the PPW. Also, note that this matrix relates the electrical behavior of the slot with the physical dimensions of the structure. Finally, the transmission matrix $\underline{\underline{T}}_{g a p / 2}$, employed in Eq. (8), is derived as the square root of the $\underline{\underline{T}}_{\text {gap }}$ matrix, exploiting the concatenation property of two transmission matrices [26].

A numerical study of $\underline{\underline{T}}_{\text {gap }}$ reveals that it has the simple form of two parallel connected impedances,

$$
\underline{\underline{T}}_{\text {series }}=\left(\begin{array}{rr}
1 & R_{\text {rad }}(\omega)-\frac{j}{\omega C_{L}(\omega)} \\
0 & 1
\end{array}\right) .
$$

This direct correspondence with lumped elements is expected, since the slot radiation losses can be modeled by the resistor, whereas the capacitor takes into account the slot capacitive behavior within the host parallel-plate waveguide as well as the field coupling to free space (reactive fields). In the next sections, it will be demonstrated that the approximation (20) is accurate, introducing very small errors. From this matrix, the radiation losses $R_{\text {rad }}(\omega)$ and series capacitor $C_{L}(\omega)$ are determined, for a particular angular frequency $(\omega)$, as

$$
\begin{aligned}
& R_{\text {rad }}(\omega)=\operatorname{Re}\left\{\mathrm{T}_{\text {gap }}(1,2)\right\}, \\
& C_{L}(\omega)=\frac{-1}{\operatorname{Im}\left\{\mathrm{T}_{\text {gap }}(1,2)\right\} \omega} .
\end{aligned}
$$

This correspondence with lumped elements also allows us to derive the complete equivalent dispersive circuit model related to the PPW CRLH LWA unit cell shown in Fig. 1 (bottom).

Finally, note that the radiation losses are only modeled by a resistor in the series branch, and that there is no radiation contribution from the shunt branch. As it is explained in [17], radiation at broadside is only achieved when the radiation losses are distributed over both the series and the shunt branches of the CRLH unit cell. Otherwise, the attenuation constant $\alpha$ tends to zero at the transition frequency. Therefore, it is expected that the type of CRLH LWAs proposed here suffers from an important drop in efficiency when radiating at broadside. However, they are still able to radiate at backward and forward directions, using the fundamental harmonic $(\nu=$ 0 ). Note that although there is a drop in the broadside radiation efficiency, the CRLH TL is still balanced. This means that the propagation constant does not exhibit a bandgap around the transition frequency.

\section{Validation Against Full-Wave Simulations}

In this section, we present a complete validation of the modal analysis of the equivalent radiating structure (see
Fig. 3). For this purpose, let us consider this structure with dimensions $\ell_{u c}=23.54 \mathrm{~mm}, g=0.5 \mathrm{~mm}, t=3.65 \mathrm{~mm}$ and $s=0.05 \mathrm{~mm}$. Note that we can chose any value for the length $\ell_{f e e d}$, because the influence of the auxiliary feeding PPW ports is removed in the analysis. For intermediate calculations, we usually set $\ell_{\text {feed }}=\ell_{u c}$. For a complete validation of the technique, the scattering parameters $S_{11}$ and $S_{21}$ related to this structure are computed for all possible phase shifts, using the proposed modal approach [Eq. (18)], and are then compared with the commercial software Ansoft HFSS (see Fig. 7). As can be observed in the figure, an excellent agreement is obtained in all cases. Also, note that the modal technique needs about 35 minutes to perform the proposed analysis, while fullwave simulations spend more than one day to obtain the same results.

\section{ITERATIVELY REFINED APPROACH FOR COMPLEX Propagation CONSTANT DETERMinATION}

In the previous sections we have explained how to compute the CRLH unit cell complex propagation constant $\left(k_{e f f}\right)$ as a function of the transmission matrix $\underline{\underline{T}}_{\text {gap }}$, and how to compute this matrix as a function of the physical dimensions of the structure and of the CRLH unit cell complex propagation constant $\left(k_{e f f}\right)$. Therefore, one can easily realize that these variables are closely inter-dependent.

In order to determine the equivalent circuit elements of the CRLH LWA unit cell [see Fig. 1 (bottom)], from previously known physical dimensions, an iterative algorithm is proposed. The description of the algorithm flow-chart, shown in Fig. 8, is as follows: initially, the non-dispersive elements of the circuit model and the CRLH transition frequency are obtained using the procedures described in section III. After that, an initial value of zero is assumed for the complex propagation constant $k_{e f f}$ at all frequencies. The transmission matrix $\underline{\underline{T}}_{\text {gap }}$ is then derived employing the proposed modal analysis, taking into account $k_{e f f}$ and the physical dimensions of the structure. Once this matrix has been obtained, the value of $k_{e f f}$ is computed based on the current value of $\underline{\underline{T}}_{g a p}$. This procedure is repeated until convergence is reached. This iterative algorithm leads to an accurate model of the slot, through the matrix $\underline{\underline{T}}_{\text {gap }}$, and to a final complex propagation constant $k_{\text {eff }}$. In the last step, the frequency dependent values of $R_{\text {rad }}$ and $C_{L}$ are extracted from the transmission matrix $\underline{\underline{T}}_{\text {gap }}$. In this way, all circuit parameters related to the unit cell are determined.

It is important to remark that this iterative algorithm is quickly convergent. Numerical simulations (see next section) demonstrate that 20-30 iterations are enough to achieve a relative error less than $10^{-12}$ between two consecutive steps over the whole frequency range.

\section{ANALYSIS OF 1D CRLH LWAS}

In this section, we carefully study a CRLH LWA comprising a periodically loaded parallel-plate waveguide. Specifically, we will design a balanced CRLH TL with a transition frequency set to $3.0 \mathrm{GHz}$. For this purpose, we choose a host waveguide filled by a material with relatively permittivity $\varepsilon_{r}=1.12$, 


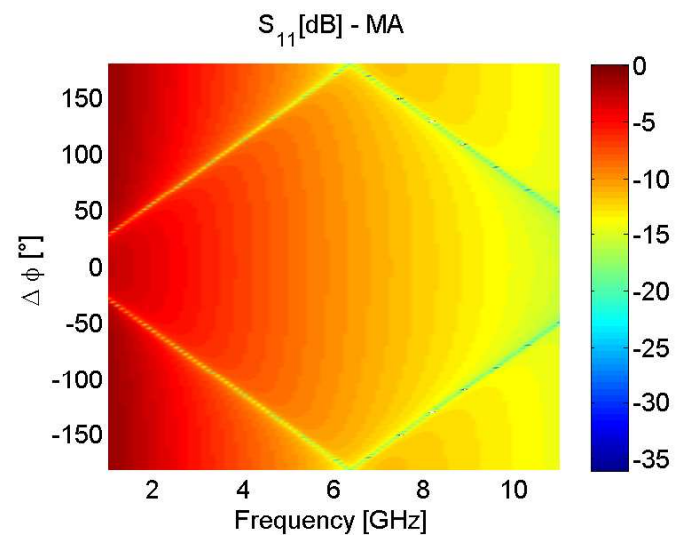

(a)

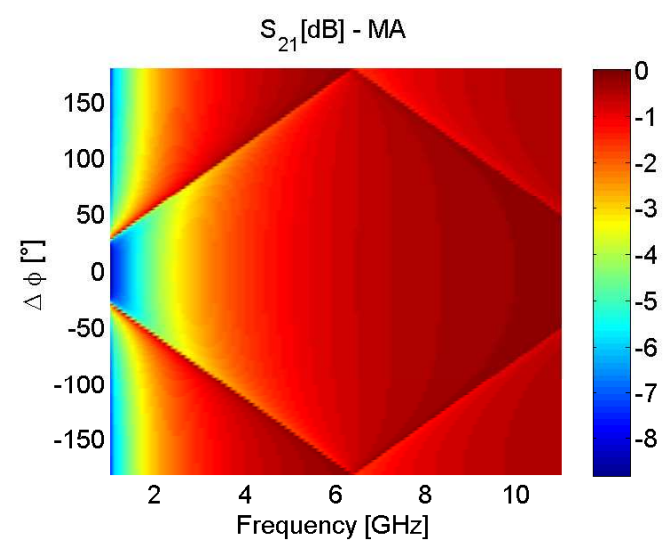

(c)

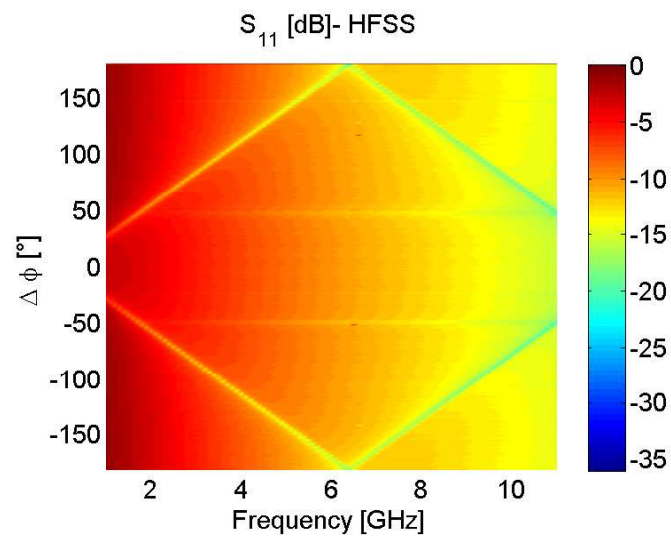

(b)

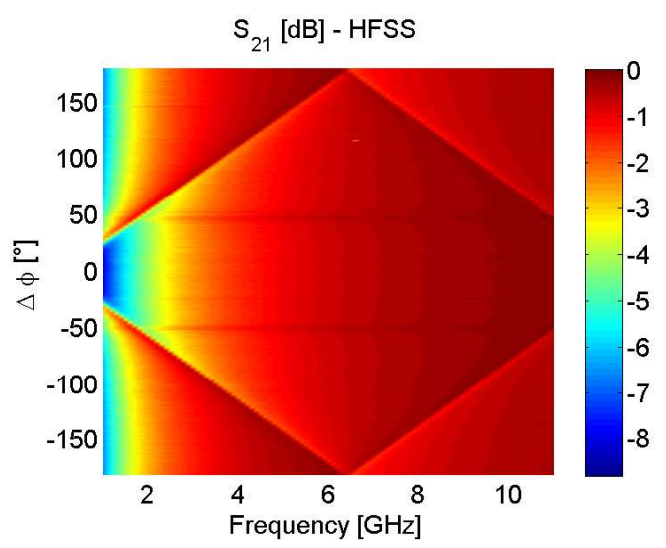

(d)

Fig. 7: Comparison of the scattering parameters $\left(S_{11}\right.$ and $\left.S_{21}\right)$ of the equivalent radiating structure (see Fig. 3) computed by HFSS and by the proposed modal analysis (MA), as a function of both, frequency and phase shift between unit cell elements. The parameters of the unit cell are $\ell_{u c}=23.54 \mathrm{~mm}, g=0.5 \mathrm{~mm}, t=3.65 \mathrm{~mm}$, and $s=0.05 \mathrm{~mm}$.

loaded by via-holes with diameter $d_{v i a}=1.0 \mathrm{~mm}$. The unit cell's total length is set to $\ell_{u c}=23.54 \mathrm{~mm}$.

The analysis steps are as follows: first, in section VI-A we will derive the waveguide's and slot's physical dimensions required to obtain a balanced design. Second, in section VI-B we rigorously analyze a single unit cell, obtaining its associated complex propagation constant (including radiation losses), and we will validate the result using HFSS. Furthermore, it is numerically demonstrated that the approximation employed to extract the frequency-dependent elements $R_{\text {rad }}$ and $C_{L}$ is accurate. Finally, in section VI-C a complete CRLH LWA composed of ten unit cells is satisfactorily analyzed using the proposed method and the results are validated using full-wave simulations.

\section{A. Balancing the CRLH unit cell}

In the case of a balanced unit cell, its associated phase constant must be equal to zero at the transition frequency (i.e. $\left.f_{c, W M}\right)$. This allows to obtain a CRLH unit cell with a smooth transition from the left-handed to the right-handed frequency region, avoiding the stopband which appears in the unbalanced case [1].
In order to determine the slot and waveguide physical dimensions, we apply the iterative algorithm developed in section V. First, we set some default physical dimensions. In this case, we choose a slot width of $g=0.5 \mathrm{~mm}$ and a metal thickness of $s=0.05 \mathrm{~mm}$, which approximates an infinitesimally thin metal (see Fig. 3). The value of $g$ is chosen to make the fabrication process easier. Then, the idea is to obtain the complex propagation constant at the frequency $f_{c, W M}$, for a range of waveguide heights $(t)$. From this analysis we select the value of $(t)$ which makes zero the real part of the complex propagation constant. Fig. 9(a) presents this analysis, which yields a final waveguide height of $t=3.65 \mathrm{~mm}$. This provides a balanced unit cell design. In order to indeed show that the unit cell is balanced, the procedure is repeated again, but fixing now the waveguide height to the new value $(t=3.65 \mathrm{~mm})$ and varying the slot width. The analysis result is shown in Fig. 9(b), which demonstrates that $g=0.5 \mathrm{~mm}$ is indeed the slot width which balances the CRLH unit cell for the given waveguide height $(t=3.65 \mathrm{~mm})$. This completes the CRLH unit cell balancing method.

It is important to remark that the proposed procedure is able to accurately balance the CRLH LWA unit cell, without requiring any full-wave simulations of the complete unit cell. In fact, 


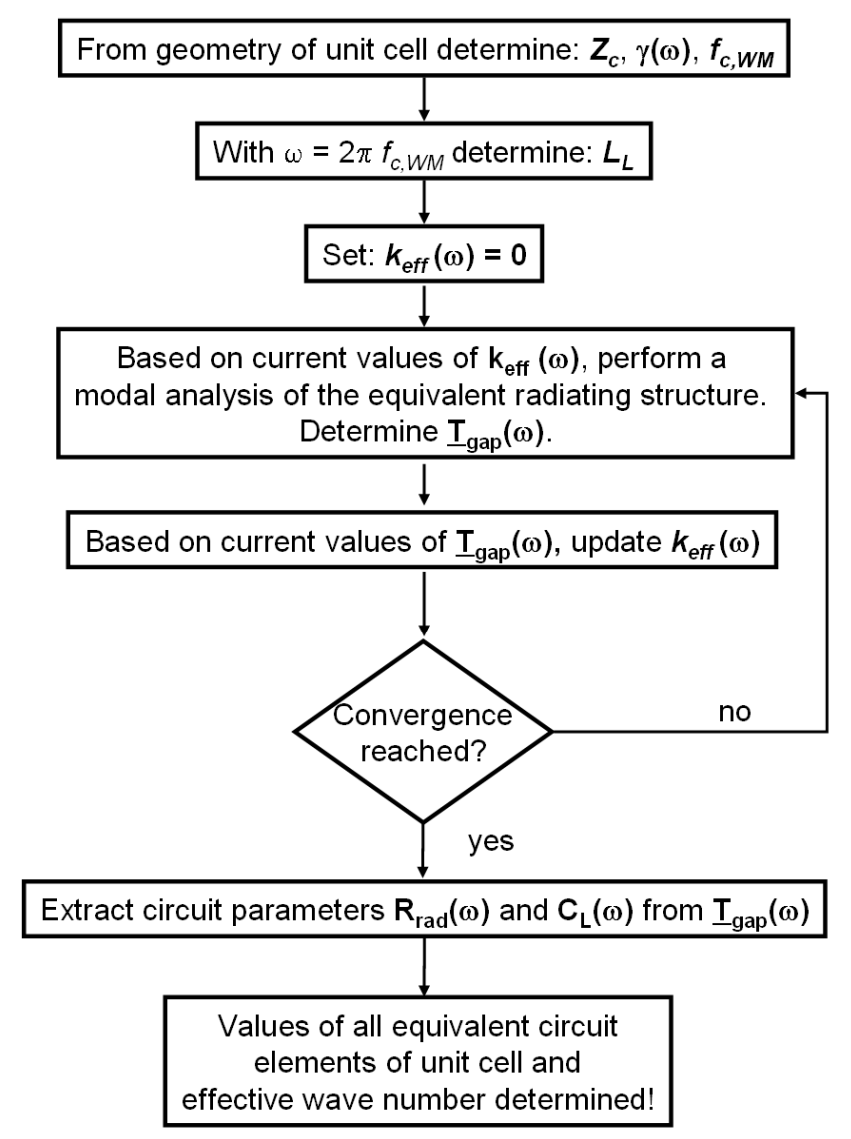

Fig. 8: Flow chart of the proposed iterative algorithm that determines the element values of the unit cell equivalent circuit [see Fig. 1 (bottom)] and the CRLH TL complex propagation constant.

with the technique proposed the modal analysis is only applied to the slot problem, and not to the complete unit cell structure. Usually, a considerable number of extremely time-consuming full-wave simulations are required to obtain a balanced-design. This is completely avoided using the proposed method, which is able to determine the physical dimensions of a balanced structure in less than 4 minutes. In addition, note that the iterative algorithm is quickly convergent, requiring just eight iterations to obtain a relative error of less than $10^{-12}$ between two consecutive steps.

\section{B. Analysis of a single CRLH unit cell}

The complex propagation constant of the CRLH unit cell is then obtained for the desired frequency region applying the iterative algorithm. A maximum of 30 iterations are required to obtain convergence (for a relative error below $10^{-12}$ between two consecutive step for all frequencies). The result of the analysis is shown in Fig. 10, for the case of iterations $i=1$ and $i=30$ (convergence reached). As it can be observed, a balanced dispersive behavior, with a transition frequency of $3.0 \mathrm{GHz}$, is clearly obtained. This is further confirmed using simulation data for the dispersion curve, which has been obtained using HFSS.

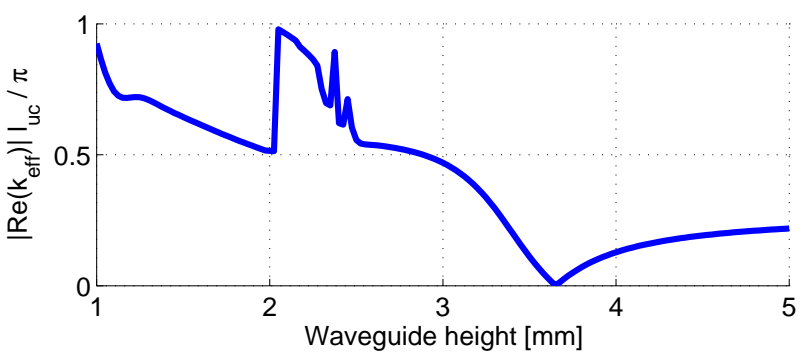

(a)

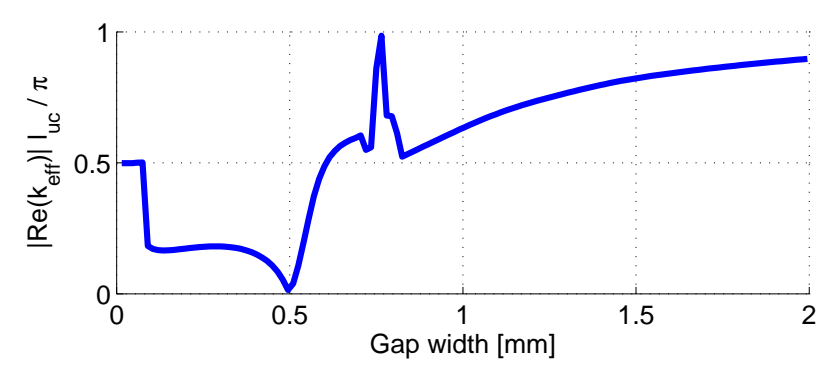

(b)

Fig. 9: Determination of the physical dimensions of the unit cell required for a balanced CRLH design, i.e. $R e\left(k_{e f f}\right)=0$. (a) Evolution of the propagation constant as a function of the waveguide height $(t)$, for a fixed value of the slot width ( $g=0.5 \mathrm{~mm}$.). (b) Evolution of the propagation constant as a function of the slot width $(g)$, for a fixed value of the waveguide height $(t=3.65 \mathrm{~mm}$.)

In Fig. 10(a) it can be observed that the dispersion curve of the $\mathrm{TM}_{2}$ mode and the $\mathrm{RH}$ parts of the $\mathrm{TM}_{1}$ and of the $\mathrm{TM}_{0}$ dispersion curve coincide very well with the real part of $k_{\text {eff }}$ for $i=30$. However, there is a discrepancy in the frequency range between $1.95 \mathrm{GHz}$ and $2.5 \mathrm{GHz}$. This is because the equivalent circuit only reproduces the propagation phenomenon of waves traveling inside the loaded PPW, while the full-wave eigenmode analysis of HFSS also considers waves which propagate in free-space above the CRLH TL. This leads to a bandgap due to the coupling between opposite waves propagating above and below the slotted surface (in the case that both types of waves are excited, which occurs in the eigenmode analysis). Note that the equivalent circuit only considers the excitation of the waves traveling inside the CRLH TL, which is correct for predicting the behavior of the proposed LWA.

In Fig. 10(b) the radiation losses of the antenna are presented. A significant decrease of the antenna efficiency at the broadside direction (i.e. at the transition frequency of the antenna) can be observed. As explained in section IV-C, this is expected for this type of unit cell configuration. In addition, the computed radiation losses accurately complete the study of the antenna radiation behavior as a function of frequency and of the physical dimensions of the structure. Usually, circuit models [1] [2] are only able to predict the phase constant, and use curve fitting to obtain a frequency-independent resistor value which models the losses. Furthermore, note that usual commercial full-wave software have also difficulties to obtain 

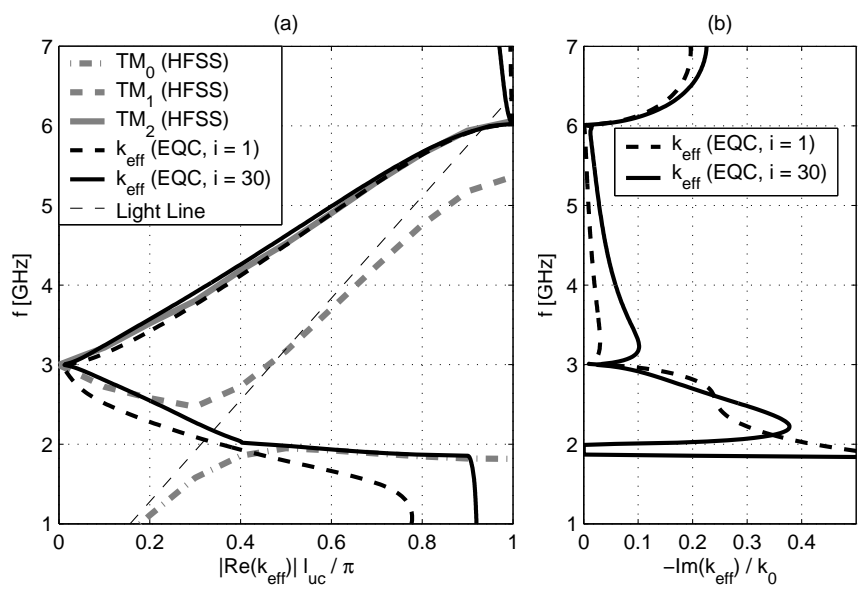

Fig. 10: Dispersive behavior of the CRLH LWA under analysis, computed with the proposed iterative algorithm after $i=1$ and $i=30$ (convergence reached) iterations. (a) Brillouin diagram, validated using HFSS. (b) Attenuation (radiation) losses versus frequency.

this parameter in infinitely periodic configurations, because they usually assume a purely real phase shift between the unit cell limits.

In order to complete the analysis, the resistor $R_{\text {rad }}(\omega)$ and capacitor $C_{L}(\omega)$, normalized with respect to the unit cell length $\ell_{u c}$, are shown as a function of frequency in Fig. 11. It is interesting to note that the bandpass frequency region of the TL (approximately from 2 to $6 \mathrm{GHz}$ ) is clearly visible in this figure. In particular, within this frequency range the capacitor exhibits smooth variations, while the value of the radiation resistance experiences a slow decrease. Also, around the lower and upper cut-off frequencies of the structure the capacitance shows an abrupt increase. This is related to the larger stored energy of the structure close to the bandpass edges, very well known in filter theory [27].

In addition, note that although the radiation losses decrease at the broadside transition (see Fig. 10(b), at $3 \mathrm{GHz}$ ) this does not correspond to a decrease of the dispersive lumped resistor value $R_{\text {rad }}$. This is due to the complex relationship between these two quantities, as explained in section III. Furthermore, note that the approximation employed to obtain the dispersive lumped parameters is very accurate. This is demonstrated in two different ways. First, the complex propagation constant obtained using $\underline{\underline{T}}_{\text {series }}$ [i.e. only with the circuit elements of Fig. 1 (bottom)] directly superimpose the full-wave results presented in Fig. 10. Second, the maximum absolute error of the terms $\underline{T}_{\text {gap }}(1,1), \underline{T}_{\text {gap }}(2,2)$, and $\underline{T}_{\text {gap }}(2,1)$ as compared with the same elements of $\underline{\underline{T}}$ series is very small, as can be observed in Fig. 12. This confirms that the proposed dispersive equivalent circuit is indeed accurate.

\section{Analysis of a ten unit cells CRLH LWA}

Finally, a single strip of width $w_{u c}$ of a complete CRLH LWA consisting of $N_{u c}=10$ identical unit cells with $g=0.5 \mathrm{~mm}$ is analyzed combining the single unit cell results obtained in the previous subsection with an ABCD

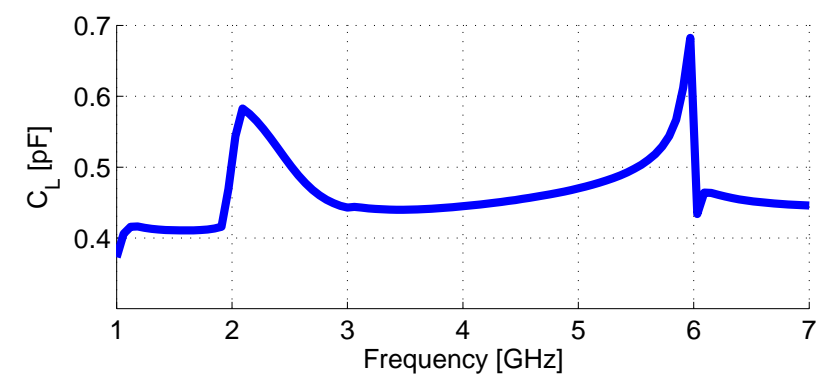

(a)

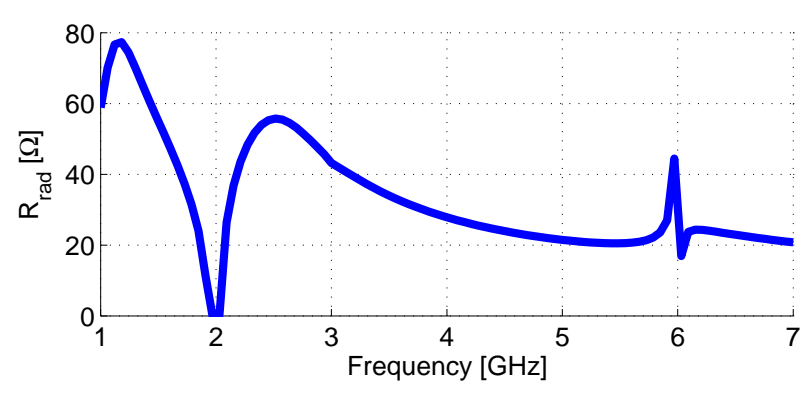

(b)

Fig. 11: Frequency dependent behavior of the dispersive lumped components shown in Fig. 1 (bottom), calculated for the CRLH unit cell described in section VI-B. (a) Series capacitor $C_{L}(\omega)$. (b) Series resistor $R_{\text {rad }}(\omega)$.

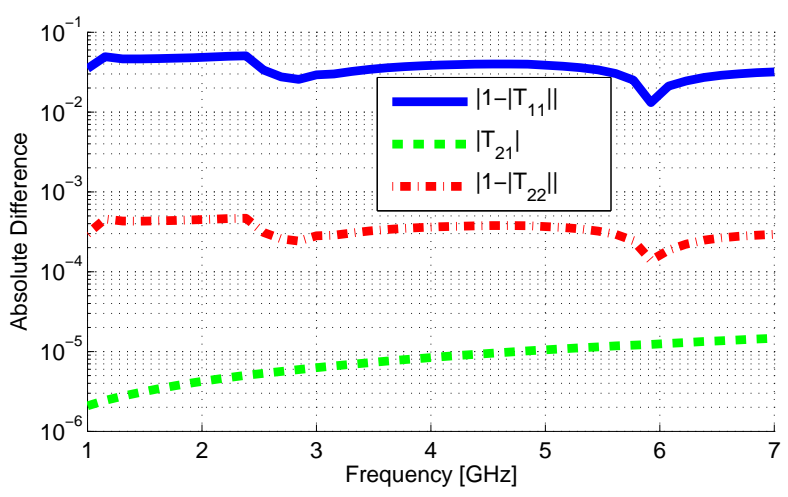

Fig. 12: Maximum absolute error of the $\mathrm{T}$ matrix elements of the equivalent radiating structure (see Fig. 3) with respect to the ideal $\mathrm{T}$ matrix related to the equivalent circuit (where $T_{11}=T_{22}=1$ and $T_{12}=0$ ), as a function of frequency.

matrix approach [1]. In order to correctly match the antenna, the width of the first and last slots $\left(g_{0}\right)$ must accurately be derived. The goal is to obtain a $g_{0}$ width which behaves as a half of slot in the infinite array environment. In this way, the first and last unit-cell of the antenna rigourously follows the equivalent circuit model of Fig. 1, and they see the PPW as a kind of continuation of the periodic structure. This leads to a smooth transition from the start/end of the CRLH structure and the unperturbed PPW within the whole frequency range. Following this strategy, the first and last slot present a capacitive behavior close to $2 C_{L}(\omega)$. In order to obtain the $g_{0}$ value, a parametric sweep for the slot, as a function of its width, is performed in the equivalent radiating 


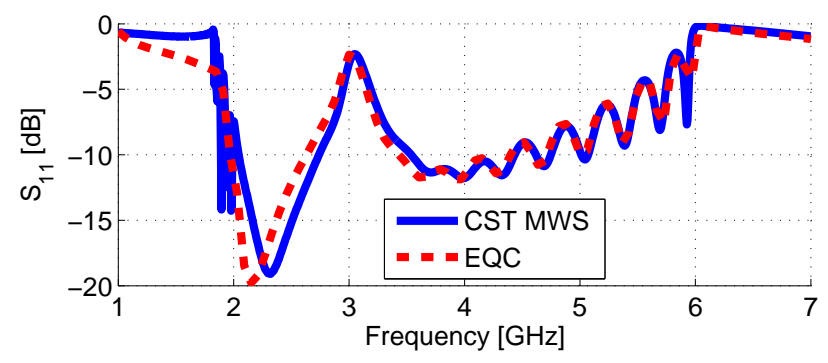

(a)

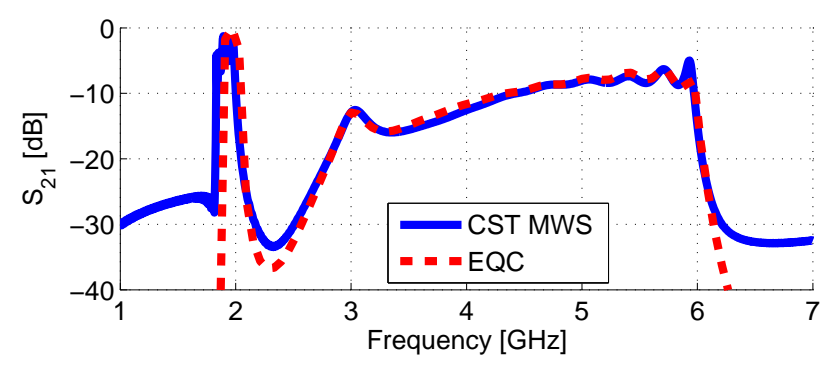

(b)

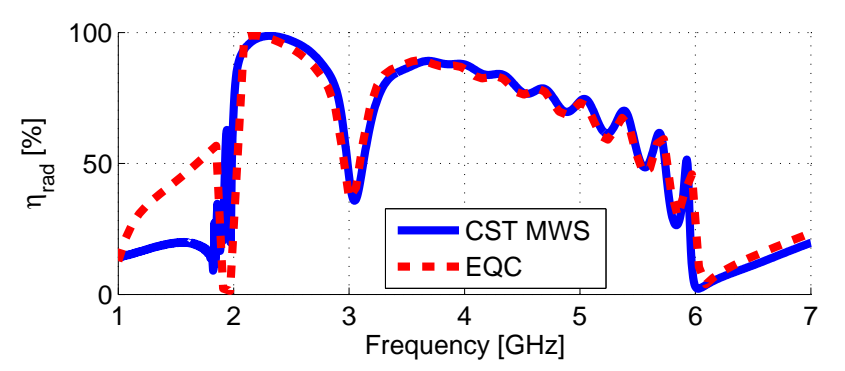

(c)

Fig. 13: Comparison of scattering parameters and radiation efficiency computed by CST Microwave Studio (CST MWST) and by the proposed iterative circuit method (EQC) of a single strip CRLH LWA consisting of ten identical reference unit cells.

structure. The approximate value found using this procedure is $g_{0}=0.157 \mathrm{~mm}$. Furthermore, note that $g_{0}$ is responsible for the connection of the CRLH TL to the feeding TL, but it does not influence the propagation characteristic of the line. Reference results for the antenna under analysis have been obtained by full-wave time domain simulation using CST Microwave Studio (MWST). The full-wave model was made up of a strip with perfectly magnetically conducting (PMC) boundary conditions applied to the lateral walls of the simulation volume, in order to represent a laterally periodic structure of infinite extension.

The magnitude of the computed scattering parameters $S_{11}$ and $S_{21}$ and the radiation efficiency $\eta_{\text {rad }}=1-\left|S_{11}\right|^{2}-\left|S_{21}\right|^{2}$ (only lossless materials were considered) are plotted in Fig. 13. As it can be observed in the figure, a very good agreement between the proposed method and the full-wave simulation is obtained. Furthermore, Fig. 14 presents the scanning capabilities of the antenna, as a function of the operating frequency. Specifically, a scanning of the main lobe from the radiation angle $\theta=-45^{\circ}$ degrees up to $\theta=+60^{\circ}$ degrees is shown.

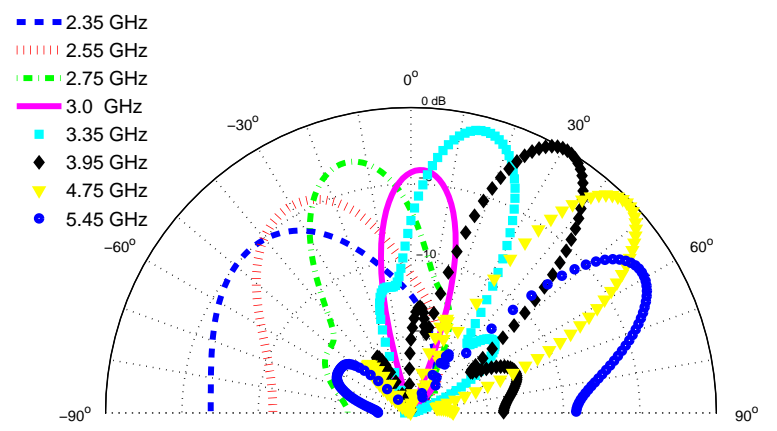

Fig. 14: Radiation pattern of the proposed CRLH LWA at different operating frequencies, showing the space scanning capabilities of the antenna.

As expected, a decrease in the radiation efficiency is found at the broadside direction $\left(\theta=0^{\circ}\right)$. Note that the directivity is higher in the $\mathrm{RH}$ region $\left(\theta>0^{\circ}\right)$ than in the $\mathrm{LH}$ frequency region $\left(\theta<0^{\circ}\right)$. This is related to the fact that the radiation losses $[\alpha(\omega)]$ are high in the LH region (as shown in Fig. 10b). Therefore, the input power is radiated in a few unit-cells, leading to a reduced effective length of the antenna (and therefore, a lower directivity). On the other hand, radiation losses are lower at the RH region, and the power is radiated along the whole structure, leading to a larger effective length of the antenna (and, consequently, to a higher directivity).

The above full-wave validations demonstrate that the proposed iterative method is able to efficiently and rigorously analyze PPW CRLH LWAs, taking into account the real physical dimensions of the structure. Furthermore, the proposed method is able to perform the analysis in just six minutes, instead of eight hours required by the full-wave simulations. This allows the application of the proposed modal-based iterative method in the analysis of practical antennas, or even to include this technique into a CAD tool for the analysis, design, and optimization of mushroom based CRLH LWAs.

\section{CONCLUSIONS}

This contribution has presented a novel modal-based iterative circuit model for the calculation of the complex propagation constant related to mushroom-like parallel-plate waveguide composite right/left-handed leaky-wave antennas (PPW CRLH LWAs). The conventional lossless CRLH unit cell configuration has been modified, including an equivalent circuit which takes into account the structure's coupling to free-space. This coupling has been modeled employing a unit cell equivalent radiating structure, which is rigorously analyzed using a multi-mode approach combined with Floquet's theorem. The resulting transmission matrix has accurately been represented by lumped elements, leading to a frequencydependent unit cell model. Then, a quickly-converging iterative algorithm has been employed to determine the final element values of the unit cell. The proposed technique was found to be accurate, and it can take into account the structure physical dimensions. The technique also allows to obtain a balanced CRLH unit cell design, it is much faster than fullwave simulations, and it provides a deep insight into the 
physics of the antenna's radiation mechanism. An antenna prototype, designed with the proposed modal-based method, is currently being manufactured and tested, and it will be presented and discussed in a future report.

\section{ACKNOWLEDGEMENT}

The authors would like to thank their colleagues $\mathrm{Mr}$. Claudius Löcker and Dr. Thomas Vaupel for many fruitful discussions. This work was partially supported by the Spanish Ministry of Education and Science under Grant FPU-AP2006015 and with the Project TEC2007-67630-C03-02.

\section{REFERENCES}

[1] C. Caloz and T. Itoh, Electromagnetic Metamaterials: Transmission Line Theory and Microwave Applications. New Jersey: Wiley-Interscience, 2006.

[2] G. V. Eleftheriades and K. G. Balmain, Eds., Negative-Refraction Metamaterials: Fundamental Principles and Applications. Hoboken, NJ: Wiley \& IEEE Press, 2005.

[3] A. A. Oliner and D. R. Jackson, "Leaky-wave antennas," in Antenna Engineering Handbook, 4th ed., J. L. Volakis, Ed. New York: McGrawHill, 2007.

[4] L. Liu, C. Caloz, and T. Itoh, "Dominant mode leaky-wave antenna with backfire-to-endfire scanning capability," Electron. Lett., vol. 38, no. 23, pp. 1414-1416, Nov. 2002.

[5] S. Lim, C. Caloz, and T. Itoh, "A reflecto-directive system using a composite right/left-handed (CRLH) leaky-wave antenna and heterodyne mixing," IEEE Microwave and Wireless Components Letters, vol. 14, no. 4, pp. 183-185, April 2004.

[6] A. Grbic and G. V. Eleftheriades, "Leaky CPW-based slot antenna arrays for millimeter-wave applications," IEEE Transactions on Antennas and Propagation, vol. 50, no. 11, pp. 1494-1504, Nov. 2002.

[7] M. A. Antoniades and G. V. Eleftheriades, "A CPS leaky-wave antenna with reduced beam squinting using NRI-TL metamaterials," IEEE Transactions on Antennas and Propagation, vol. 56, no. 3, pp. 708-721, March 2008.

[8] D. Sievenpiper, L. Zhang, R. F. Jimenez Broas, N. G. Alexópolous, and E. Yablonovitch, "High-impedance electromagnetic surfaces with a forbidden frequency band," IEEE Transactions on Microwave Theory and Techniques, vol. 47, no. 11, pp. 2059-2074, Nov. 1999.

[9] D. Sievenpiper, J. Schaffner, J. J. Lee, and S. Livingston, "A steerable leaky-wave antenna using a tunable impedance ground plane," IEEE Antennas and Wireless Propagation Letters, vol. 1, pp. 179-182, 2002.

[10] C. A. Allen, C. Caloz, and T. Itoh, "Leaky-waves in a metamaterialbased two-dimensional structure for a conical beam antenna application," in IEEE MTT-S Int. Microwave Symp. Dig., vol. 1, June 2004, pp. 6-11.

[11] T. Bertuch, "A TM leaky-wave antenna comprising a textured surface," in Proc. Int. Conf. Electromag. Adv. Appl. (ICEAA), Sept. 2007.

[12] I. J. Bahl and K. C. Gupta, "A leaky-wave antenna using an artificial dielectric medium," IEEE Transactions on Antennas and Propagation, vol. 22, no. 1, pp. 119-122, Jan. 1974.

[13] _ - "Frequency scanning by leaky-wave antennas using artificial dielectrics," IEEE Transactions on Antennas and Propagation, vol. 23, no. 4, pp. 584-589, July 1975.

[14] _ "Radiation from a dielectric-artificial dielectric slab," IEEE Transactions on Antennas and Propagation, vol. 24, no. 1, pp. 73-76, Jan. 1976.

[15] I. J. Bahl and P. Bhartia, "Leaky-wave antennas using artificial dielectrics at millimiter wave frequencies," in IEEE Transactions on Antennas and Propagation, vol. 18, June 1980, pp. 23-26.

[16] R. J. King, D. V. Thiel, and K. S. Park, "The synthesis of surface reactance using an artificial dielectric," IEEE Transactions on Antennas and Propagation, vol. 31, no. 3, pp. 471 - 476, May 1983.

[17] S. Paulotto, P. Baccarelli, F. Frezza, and D. R. Jackson, "Full-wave modal dispersion analysis and broadside optimization for a class of microstrip crlh leaky-wave antennas," IEEE Transactions on Microwave Theory and Techniques, vol. 56, no. 12, pp. 2826 - 2837, December 2008.

[18] C. S. Paulotto, P. Baccarelli, F. Frezza, and D. R. Jackson, "A novel technique for open-stopband suppression in 1-d periodic printed leakywave antennas," IEEE Transactions on Antennas and Propagation, vol. 57, no. 7, pp. 1894-1906, July 2009.
[19] S. Marini, A. Coves, V. Boria, and B. Gimeno, "Efficient Modal Analysis of Periodic Structures Loaded With Arbitrarily Shaped Waveguides," IEEE Transactions on Microwave Theory and Techniques, vol. 58, no. 3, pp. 529-536, March 2010.

[20] R. F. Harrington, Time-Harmonic Electromagnetic Fields. New York: McGraw-Hill, 1961.

[21] D. Pozar, Microwave Engineering. 3rd Edition. New Jersey: John Wiley and Sons, 2005.

[22] F. Arndt, I. Ahrents, U. Papziner, U. Wiechmann, and R. Wilkeit, "Optimized e-plane t-junction series power dividers," IEEE Transactions on Microwave Theory and Techniques, vol. 35, no. 11, pp. 1052 - 1059, November 1987.

[23] K. H. Park, H. J. Eom, and Y. Yamaguchi, "An analytic series solution for e-plane t-junction in parallel-plate waveguide," IEEE Transactions on Microwave Theory and Techniques, vol. 42, no. 2, pp. 356 - 358, February 1994.

[24] Y. H. Cho, "New iterative equations for an e-plane t-junction in a parallel-plate waveguide using green's functions," Microwave and Optical Technology Letters, vol. 37, no. 7, pp. 447 - 449, June 2003.

[25] N. Marcuvitz, Waveguide Handbook. Boston, Massachusetts, USA: MIT Radiation Laboratory Series, 1964.

[26] D. M. Pozar, "Analysis and design of cavity coupled microstrip couplers and transitions," IEEE Transactions on Microwave Theory and Techniques, vol. 51, no. 3, pp. 1034-1044, March 2003.

[27] C. Ernst and V. Postoyalko, "Prediction of peak internal fields in directcoupled-cavity filters," IEEE Transactions on Microwave Theory and Techniques, vol. 51, no. 1, pp. 64-73, January 2003. 